\title{
Implementación de estrategias pedagógicas constructivistas mediadas por las herramientas Web 2.0 para el fortalecimiento de la comprensión teórica en los contenidos conceptuales de las ciencias naturales y la educación ambiental
}

\author{
Emerson Leonardo Tovar Vergara
}

Recibido: 18-08-2018

Aceptado: 03-02-2019

\section{Resumen}

La investigación tiene como objetivo implementar estrategias pedagógicas constructivistas mediadas por las herramientas Web 2.0 para el fortalecimiento de la comprensión teórica en los contenidos conceptuales de la asignatura de ciencias naturales y la educación ambiental, en los estudiantes del grado octavo del I.E. Técnico Politécnico, Bucaramanga. Las estrategias pedagógicas se presentaron a través de cuatro unidades temáticas sobre las células, en ella se realizaron talleres, mapas mentales, cuadros sinópticos, sopas de letras entre otras actividades que se desarrollarán en el proyecto de aula dando solución a las falencias en los estudiantes. Los conceptos que permitieron comprender y argumentar el proceso investigativo fueron el constructivismo, la metodología de la enseñanza y aprendizaje y el aprendizaje significativo. La población objeto de estudio estuvo conformada por 145 estudiantes perteneciente a la I.E. Técnico Politécnico, de ella se extrajo una muestra intencional de 35 estudiantes que conforman

1. Facultad de educación. Universidad Autónoma de Bucaramanga. Magister en educación.

Bucaramanga - Colombia.

Correo electrónico: emletovar@hotmail.com

ORCID: http://orcid.org/0000-0002-1594-9409 
el grado de octavo de la misma institución educativa. De aquí que la metodología que se llevó a cabo consistió en la investigación acción, con diseño no experimental de tipo descriptivo. Las técnicas de recolección de datos fueron el análisis documental, la observación, y las rúbricas de evaluación, los cuales evidenciaron los resultados que se centraron en el fortalecimiento de la comprensión teórica a través de la implementación de estrategias pedagógicas constructivista para la enseñanza y aprendizaje en los contenidos conceptuales en el área de ciencias naturales y educación ambiental, las cuales facilitaron ampliar la construcción de aprendizajes significativos.

Palabras clave: comprensión teórica, contenidos conceptuales, ciencias naturales, aprendizaje, constructivismo.

\title{
Implementation of constructivist pedagogical strategies mediated by Web 2.0 tools to strengthen the theoretical understanding of the conceptual contents of the natural sciences and environmental education
}

\begin{abstract}
The research aims to implement constructive pedagogical strategies mediated by Web 2.0 tools to strengthen the theoretical understanding of the conceptual contents of the subject of natural sciences and environmental education, in the eighth grade students of the I.E. Polytechnic Technician, Bucaramanga. The pedagogical strategies are presented through four thematic units on the Cells, in which workshops, mental maps, synoptic tables, letters of letters among other activities that are developed in the classroom project,
\end{abstract}


giving solution to the shortcomings in the students. The concepts that allowed to understand and argue the investigative process were constructivism, the methodology of teaching and learning and meaningful learning. The population studied was made up of 145 students belonging to the I.E. Polytechnic Technician, from her an intentional sample of 35 students that make up the eighth grade of the same educational institution. Hence, the methodology that was carried out consisted of action research, with a non-experimental design of a descriptive type. The data collection techniques were documentary analysis, observation and evaluation norms, which showed the results that focused on the understanding of the constructive pedagogical applications for teaching and learning. The conceptual contents in the area of natural sciences and environmental education, the measures to expand the construction of significant learning.

Keywords: theoretical understanding, conceptual contents, natural sciences, learning, constructivism.

\section{Introducción}

La didáctica de las ciencias naturales y la educación ambiental a nivel internacional tiene su auge especialmente en Europa y Estados unidos hace unos 30 años, en donde comienza a emerger como una disciplina independiente. Leymonié (2009), expresa que las primeras reformas curriculares de ciencias se centraban en modificar el enfoque tradicional en donde la práctica y la experimentación estaba totalmente ausente de las aulas y se basaba únicamente en la enseñanza por transmisión del conocimiento, por lo tanto, el papel del docente era primordial y lo único que se esperaba del alumno era la asimilación de los contenidos conceptuales suministrados por el docente. 
Aunado a esto, la enseñanza y aprendizaje de las diferentes áreas del conocimiento han requerido de la habilidad de comprensión cognitiva, de los estilos de aprendizaje y de la manera de enseñar las teorías. En la historia de la sociedad desde el inicio de la escritura ha sido importante la lectura y el análisis de los mensajes que pueden ser gráficos o letras, ya que, indudablemente, cuando el individuo logra una comprensión lectora óptima se les facilita contar con mejores oportunidades educativas, laborales y sociales (Ospina, 2010); en consecuencia las investigaciones que se puedan realizar al respecto tienen como propósito fundamental propiciar acciones pedagógicas que mejoren el nivel lector de las personas.

En consecuencia, el objetivo perseguido por estas reformas expresadas por Leymonié (2009), era la incorporación de nuevas metodologías didácticas en donde le permitieran al estudiante proponer interrogantes o "aprender haciendo". Por su parte, Piaget (1976), en su teoría determina que el pensamiento formal es condición no sólo necesaria sino suficiente para acceder al conocimiento científico y ayudar a desarrollar estructuras fundamentales en el individuo independientemente de los contenidos para que sea capaz de comprender cualquier concepto científico; las posturas más radicales en esta línea conciben que todas las disciplinas deberían encaminarse a enseñar a pensar formalmente, con independencia del contenido; es decir, a dominar el método científico en los procesos de la Ciencia.

En este sentido, la Organización de las Naciones Unidas para la Educación, la Ciencia y la Cultura, UNESCO (2008), señala la importancia de la enseñanza de las Ciencias Naturales en la educación, la cual contribuye a la formación del pensamiento cognitivo para dar respuestas especificas a través de la resolución de problemas concretos, es decir mediante el conocimiento procedimental. Por otra parte, esta disciplina mejora la calidad de vida, prepara a los estudiantes para la futura inserción en el mundo científico y tecnológico, promueve el desarrollo intelectual, sirve de soporte y sustrato de aplicación para las áreas instrumentales, permite la exploración lógica y sistemática del ambiente y explica la realidad y ayuda a resolver problemas que tienen que ver con ella. 
Debido a los avances tecnológicos de los últimos años, los cuales han producido un fuerte impacto en la educación, según, García (Pósito, 2012) amplían los escenarios educativos, en donde los campus físicos se reemplazan o se complementan con otros de carácter virtual. En consecuencia, para efectos de esta investigación la comprensión teórica parte de la base que leer es un acto comunicativo, cobra importancia "el aprendizaje lector a través de las secuencias textuales, ya que, el ser humano puede comunicarse de diferentes formas y estilos y dentro de un mismo texto pueden haber muchas tipologías" (Del Moral, 2010) donde el lector debe saber relacionarse con ellas para entender lo escrito y poder hacer inferencias acertadas que buscan formar una persona con un alto rendimiento lector dentro de la sociedad.

Es importante mencionar que, a nivel latinoamericano, Chile es uno de los países con mejor desempeño en las pruebas internacionales que miden competencias científicas en estudiantes de enseñanza básica y media, según, Martin, González \& Chrostowski (2003), mencionan que no solo es necesario saber cómo es la forma adecuada de enseñar ciencias, sino que también existan las condiciones necesarias para poder efectuar la indagación científica con los estudiantes. (citado por Cofré, Camacho, Galaz, Jiménez Santibáñez \& Vergara, 2010)

Cofré et al (2010), resalta que los docentes de ciencias naturales deben contar con habilidades, conocimientos y aptitudes especificas las cuales permiten evaluar el conocimiento teórico y práctico mediante la aplicación de una didáctica de las ciencias efectiva, el dominio de la disciplina, el Manejo del currículo y de diferentes metodologías de evaluación, la generación de una relación de confianza y respeto con los alumnos, la capacidad de reflexión de su práctica y la actualización permanente y manejo de nuevas tecnologías.

Por otra parte, en el contexto nacional en el año 2006 Colombia participó por primera vez en el estudio PISA, año en el cual se profundizó en el área de ciencias. En 2009, el énfasis fue el área de lectura y en 2012 fue matemáticas. Para PISA 2015, el área de ciencias volvió a ser el enfoque principal de la prueba. 
Es evidente que, desde su primera participación en 2006, Colombia ha mejorado notablemente su desempeño en las tres áreas evaluadas. El área de lectura es donde se observa el mayor progreso: en 2015 obtuvo 40 puntos más en el puntaje promedio, en comparación con el resultado de 2006. Mientras que, durante este período, en matemáticas y ciencias aumentamos 20 y 28 puntos, respectivamente (Ministerio de educación Nacional, 2015).

Sin embargo, es notorio que las Ciencias Naturales y la Educación Ambiental no son asignaturas complejas, pero requieren de una mayor comprensión para asimilar sus contenidos y la importancia que tienen para la cotidianidad. Por ello, se ha observado que la falta de comprensión teórica se ha convertido en un problema crucial en las instituciones educativas, no solo de Colombia sino también de sus alrededores, tal y como se observa en las pruebas internacionales de PISA.

Así mismo, el Ministerio de Educación Nacional (2015, p. 6), señala que existen debilidades en los estudiantes frente al desarrollo de capacidades conceptuales como: reconstruir teorías, formular hipótesis, diseñar experimentos, argumentar, imaginar, ser creativos y construir alternativas de solución a problemas del entorno, los cuales son objetivos reiterados en los lineamientos curriculares y estándares de competencias.

Al respecto, Castro y Ramírez (2013, p. 17), mencionan que para el caso de las Ciencias Naturales y la Educación Ambiental es importante crear espacios que permitan la ejecución de actividades en el aula en donde el estudiante logre descubrir los conocimientos a través del contacto con la realidad involucrando actividades de observación, enunciando hipótesis, y la experimentación y formulación de conceptos. Desde esta perspectiva, los cambios en la enseñanza de las Ciencias Naturales, responden a las necesidades actuales de la sociedad, en donde los individuos deben poseer ciertas competencias científicas $y$, además, poder desarrollar habilidades lógicas de pensamiento; por ende, estar informados y capacitados, lo cual permite apropiarse de los nuevos contenidos de los diferentes campos conceptuales y comprender mejor la realidad. 
En este sentido Hermosa Del vasto (2015), describe que el radical impacto de la información tecnológica, la globalización y el crecimiento acelerado de las economías ha dado como resultado que los estudiantes aprenden, representan y utilizan el saber de diferentes modos y con una variedad de medios para resolver problemas y transformar la educación. Por tal motivo, la mayoría de los países desarrollados hacen grandes esfuerzos por atender las demandas de una nueva educación, a través del uso de las TIC.

Por lo tanto, Rodiño (2014), afirma que existen diversas dificultades en el proceso de enseñanza aprendizaje de la educación ambiental y las ciencias naturales tales como, desmotivación y falta de interés las cuales van creciendo dependiendo del contexto; estas dificultades se intensifican en los contenidos del área de ciencias principalmente, ya que estas demandan un esfuerzo adicional por parte del estudiante. Por otra parte, el mismo autor señala que, se ha evidenciado el creciente interés de los jóvenes por el uso de celulares, tabletas, computadores y en general diferentes medios electrónicos y sus respectivas aplicaciones, dichas herramientas utilizadas de una forma adecuada y en supervisión por el cuerpo docente y padres de familia, pueden ser de gran ayuda en la transmisión de los contenidos teóricos científicos y facilitar su entendimiento e interpretación, así como también despertar en los estudiantes el agrado por la asignatura.

Por tal motivo, el gobierno nacional con el ánimo de mejorar la calidad de la educación ejecuta inversiones significativas en la incorporación e implementación de herramientas tecnológicas que enriquezcan el proceso de enseñanza y aprendizaje (Aparicio, 2018); dentro de sus objetivos se encuentra el de garantizar el acceso, permanencia y condiciones favorables de los estudiantes en instituciones de calidad, mejorando el desarrollo de competencias y habilidades, para tal fin se invierte en equipos de cómputo con acceso a Internet, tal como lo indica, Marín y Donoso (2014), con el programa "Tablet para educar, el más reciente aporte del gobierno a este gran avance"

Una vez detectado la problemática, es importante mencionar que la presente investigación se realiza en el Instituto Politécnico- 
Bucaramanga con estudiantes del grado octavo en donde se evidencia un bajo desempeño en el área de ciencias naturales incidido por la falta de comprensión lectora, como se observa en el siguiente gráfico el cual muestra los resultados de las pruebas Saber del año 2016 aplicadas a estudiantes del grado noveno de la misma institución.

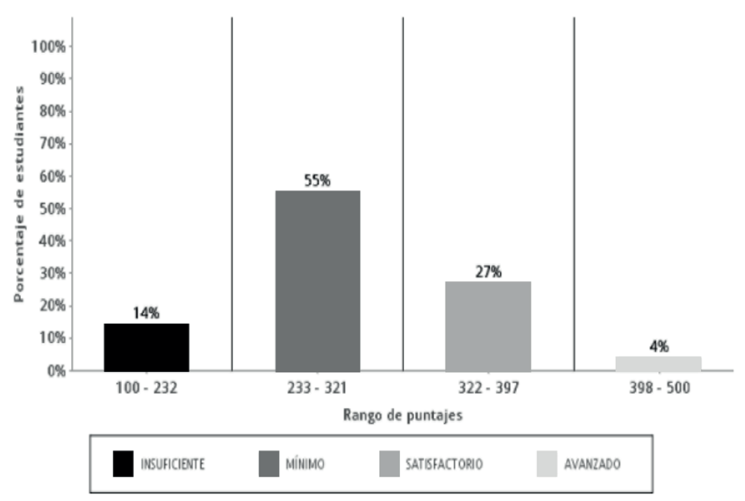

Gráfico 1. Porcentaje de estudiantes por niveles de desempeño en el establecimiento educativo (Ciencias naturales - grado noveno). Fuente. Elaboración propia

El gráfico, muestra el desempeño de los estudiantes de dicha institución donde el $55 \%$ de los estudiantes se encuentran ubicados en el nivel de desempeño mínimo y resaltando que no sólo se trata de un problema con los educandos, sino también de la metodología de enseñanza-aprendizaje que se han utilizado en las ciencias naturales y la educación ambiental en el proceso curricular, se evidencia que se necesita urgentemente de estrategias pedagógicas que propicien y fortalezcan el proceso de enseñanza-aprendizaje de los estudiantes de octavo grado de la institución a través del aprovechamiento de las herramientas web 2.0, que se componga de medios digitales que provean de más dinamismo y modernidad a las clases.

En consecuencia, el Instituto Politécnico de Bucaramanga presenta un bajo rendimiento académico en la asignatura de Ciencias Naturales y Educación Ambiental causado por el bajo desempeño de la comprensión teórica en algunos contenidos conceptuales, por esta razón surge la necesidad de implementar estrategias peda- 
gógicas constructivistas, mediadas por las herramientas web 2.0, para el fortalecimiento de la compresión lectora en los contenidos conceptuales de las Ciencias Naturales y la Educación Ambiental en los estudiantes de octavo grado, con el fin de que estos estudiantes no solo mejoren el rendimiento académico, sino, concientizar a los estudiantes sobre la importancia que tienen las Ciencias Naturales y la Educación Ambiental desde el contexto educativo.

Finalmente, y en consecuencia a lo mencionado anteriormente, surge el interrogante de ¿Cómo las estrategias pedagógicas constructivistas mediadas por las herramientas Web 2?0, contribuyen al fortalecimiento de la comprensión teórica de los contenidos conceptuales en el área de Ciencias Naturales y Educación Ambiental en estudiantes de grado octavo?

De esta forma el estudio tuvo como objetivo fortalecer la comprensión teórica en los contenidos conceptuales de las ciencias naturales y la educación ambiental en estudiantes de grado octavo del i.e. técnico politécnico de Bucaramanga mediante la implementación de estrategias pedagógicas constructivistas.

En este sentido, la investigación busca innovar en el ámbito de la tecnología educativa en donde estas herramientas no sean vistas solamente en la asignatura de tecnología e informática, sino que sean aplicadas en diferentes áreas, como es el caso de las ciencias naturales y educación ambiental, especialmente en el fortalecimiento de los contenidos teóricos y la comprensión de lectora que se ha convertido en una debilidad cognitiva para los estudiantes.

Es importante expresar que la aplicación de esta propuesta refuerza la relación docente-alumno a través de una atmósfera de aprendizaje amena y en donde los educandos se sientan conformes. Además, la comprensión teórica es una de las competencias fundamentales para el desarrollo del conocimiento en las personas, desde los primeros grados de escolaridad se va desarrollando, a partir de los procesos humanos cognitivos y meta cognitivos que promueven esta capacidad; permitiendo así el alcance de las competencias comunicativas necesarias (Barrantes, Beltrán y Pérez 2016). 
En consecuencia, la investigación contribuye a la calidad académica y educativa del plantel, puesto que un buen rendimiento académico en las Ciencias Naturales y la Educación Ambiental, implica una mejor puntuación en las pruebas externas realizadas por el ICFES, hecho que nutre de forma positiva la misión y la visión institucional.

Igualmente, Sancho (2001), menciona que es de vital importancia la complementación de la enseñanza específica y tradicional en los temas relacionados a las Ciencias Naturales, con las herramientas virtuales ofrecidas por la web 2.0, ya que el proceso de enseñanza-aprendizaje de las ciencias, tiene un alto contenido de conceptos complejos, y estas tecnologías facilitan el aprendizaje autónomo y significativo, favoreciendo un estilo docente más personalizado y participativo, y mejora el rendimiento académico de los estudiantes.

En concordancia a lo anterior, el estudio genera un impacto social tanto a la institución como al sistema educativo, debido a la importancia de adquirir nuevos conocimientos que complementen la formación académica. Además, los conocimientos adquiridos por los estudiantes durante la ejecución de la propuesta pedagógica mediadas por el uso de las herramientas Web 2.0, consolidan la educación que el programa vive digital establece.

Por otra parte, esta investigación permite que la enseñanza de las Ciencias Naturales y Educación Ambiental, como asignatura de aprendizaje elemental e indispensable en la vida cotidiana de los individuos, sea fundamental para la adquisición de los conocimientos y habilidades científicas aplicadas a distintos contextos cercanos de los estudiantes como a situaciones prácticas o de resolución de problemas, propios de la sociedad actual.

De igual manera, las actitudes científicas deben ser evaluadas por el profesor, tal y como se presenta en esta investigación, con el propósito de mejorar no solo el proceso educativo sino también el cuidado del medio ambiente. (Ministerio de Educación Nacional, 2015). 
Por medio de la aplicación de este proyecto, se pretende que los estudiantes se involucren en el aprendizaje colaborativo y significativo (de la calle, Malaver, Gallego, Rodríguez, Flórez, Saldaña, 2014), estableciendo en ellos una cultura de responsabilidad y compromiso en la formación y desarrollo de las habilidades propias para el manejo de herramientas web 2.0. Por lo tanto, mediante estas herramientas se realizan las actividades didácticas que serán desarrolladas por los estudiantes en un lapso de tiempo establecido, así mismo los temas relacionados y propuestos en el currículo escolar establecidos así por la asignatura de ciencias naturales y educación ambiental contribuyen en la formación académica y profesional de los estudiantes.

Finalmente, la presente investigación proporciona un aporte significativo para la institución en cuanto al mejoramiento del rendimiento académico de los estudiantes, especialmente en el área de Ciencias Naturales y Educación Ambiental, el cual será demostrado en las pruebas internas que se realizan durante todo el año escolar, de igual forma, incentiva la formación docente en cuanto a capacitaciones en la implementación de las TIC y uso de las herramientas web 2.0, desde su rol como formador de procesos de enseñanza-aprendizaje, ya que es el docente quien por medio de la observación conoce las debilidades, fortalezas y oportunidades de mejora, y, con ello puede diseñar e implementar propuestas pedagógicas que conlleven a superar las dificultades presentadas por los estudiantes.

\section{Metodología}

Se identificaron los antecedentes internacionales, nacionales y locales.

\section{Antecedentes Internacionales}

En este contexto, se tiene la investigacion realizada por Pósito (2012), titulada "El Problema de enseñar y aprender Ciencias Na- 
turales en los nuevos ambientes educativos", el objetivo general consistió en generar una solución tecnológica y pedagógica al problema de diseño de prácticas de aprendizaje, a través del desarrollo de una aplicación web a nivel de prototipo denominado Gestor de Prácticas de Aprendizaje, GPA. Se espera que el Gestor permita diseñar y administrar las prácticas de aprendizaje con un asistente que oriente al docente en los aspectos pedagógicos-didácticos. El prototipo denominado Gestor de prácticas de aprendizaje GPA, traduce acciones conducentes a generar diseños de prácticas de aprendizaje creativos, que promuevan la comprensión de los contenidos de las Ciencias Naturales y sean pertinentes a los nuevos ambientes educativos.

Esta tesis se centró en el problema de diseño de prácticas de aprendizaje en el área disciplinar de las Ciencias Naturales mediante la investigación descriptiva de las cualidades y dificultades que poseen los docentes al usar nuevas herramientas tecnológicas. Para los nuevos ambientes educativos y en la necesidad de ofrecer asistencia pedagógica al docente de nivel superior al momento de diseñarlas (Ortiz, Buitrago, 2017). Para responder a dicho problema se plantearon interrogantes tales como ¿Qué aspectos epistemológicos caracterizan las Ciencias Naturales? ¿Qué Prácticas de aprendizaje facilitan la mediación pedagógica y comunicacional del área del conocimiento de las Ciencias Naturales en el nivel superior? ¿Qué recursos de los nuevos ambientes facilitan la comprensión a las Ciencias Naturales? ¿Qué aspectos son necesarios tener en cuenta para diseñar una práctica de aprendizaje? ¿Qué asistencia pedagógica requiere el diseño de dichas prácticas de aprendizaje? Las respuestas a estos interrogantes indujeron a la necesidad y posibilidad de disponer de una aplicación tecnológica que asista al docente en el diseño y producción de las Prácticas de Aprendizaje, dada la importancia que éstas tienen como puentes de mediación pedagógica para acompañar y promover el aprendizaje de los alumnos.

Esta investigación deja un aporte significativo con respecto al uso de las TIC para la presente investigación, la cual representa para los docentes del área de las Ciencias Naturales, un facilitador 
tecnológico y pedagógico para diseñar prácticas de aprendizaje utilizando los recursos del ambiente educativo en forma apropiada y creativa, contribuyendo a mejorar la calidad de la educación y a reforzar la comprensión teórica en los contenidos específicos del área de las Ciencias Naturales y Educación Ambiental.

Otra de la investigación considerada de gran interés para este estudio, es la propuesta por Alcalá (2012), titulada "aplicación de un programa de habilidades meta cognitivas para mejorar la comprensión lectora en niños de cuarto grado de primaria del colegio Parroquial Santísima Cruz de Chulucanos" en Piura (Perú). La orientación de esta investigación fue primordial en conocer la influencia que tiene el desarrollo de un programa de habilidades meta cognitivas en el nivel de comprensión lectora de los niños de cuarto grado de primaria del colegio Parroquial Santísima Cruz. Su proyecto consistió en realizar un análisis de las pruebas censales del ministerio de educación peruano en el que identificaron que los resultados son nefastos por lo tanto se plantea efectuar una investigación que promueva estrategias que permitan en los alumnos fortalecer la autonomía y en especial en los de grado cuarto, ya que ellos se encuentran inmersos en el mundo de las letras (semántica estructurada) y que están relacionadas con la comprensión lectora y a su vez interactúan con la evolución de las habilidades meta cognitivas.

El muestreo y la parte cualitativa de la investigación y las técnicas e instrumentos de recolección de datos se desarrollaron de la siguiente forma: en un paradigma socio crítico que parta de la reflexión de la situación actual. La investigación se desarrolló con una metodología mixta y se implementó a los alumnos de cuarto grado a quienes se les aplicaron test, pruebas, cuestionarios de manera progresiva según sus habilidades de comprensión lectora, demostradas en las diferentes áreas del conocimiento, y con destrezas que durante su vida han desarrollado en el contexto familiar.

Al finalizar el proyecto se evidenciaron análisis estadísticos de pre-test, pos-test, en el que se señaló la falencia para dominar las habilidades de comprensión lectora solicitadas en las pruebas 
nacionales y se aplicó a un grupo control la misma prueba, la cual indicó un nivel superior, en el que se puede concluir que, aunque sea la misma institución, todos los grupos no avanzan igual.

Analizando esta investigación con el presente estudio, se deduce que la implementación de unas estrategias bien diseñadas y oportunamente aplicadas, conlleva a mejorar los resultados de las pruebas propuestas por el Estado, alcanzando niveles altos de comprensión lectora y el desarrollo cognitivo de los educandos.

Por su parte, Medina y Badilla (2013) en su investigación "Uso de ambientes virtuales en el aprendizaje de las Ciencias" resaltan que el uso innovador de la Web 2.0, motiva la construcción de un curso de Biología b-learning, basado en el Modelo Pedagógico MEDIVAL para mejorar el desarrollo de habilidades cognitivas básicas en los Contenidos Mínimos Obligatorios (CMO) de Biología que mida la PSU de Ciencias, donde las actividades didácticas se organizan sobre la base de los recursos que ofrece la plataforma Moodle. El paradigma del estudio es positivista, el cual corresponde a una investigación descriptiva de carácter cuantitativa, cuyo diseño respondió a la metodología cuasi-experimental, centrándose en el análisis de las habilidades cognitivas, que poseen 30 estudiantes del curso PSU de Ciencias de un Preuniversitario de Concepción antes y después de realizar un curso de Biología blearning.

Los resultados obtenidos a través de una encuesta señalan que el uso del ambiente virtual como complemento a las clases presenciales, incrementa en el grupo experimental el desarrollo de las habilidades cognitivas evaluadas en los cinco ejes temáticos estudiados, logrando una diferencia significativa, respecto del control (Konieczny, 2015). Además, se observan diferencias significativas en los resultados de la PSU de Ciencias en el grupo control y experimental. Los ambientes virtuales pueden conformarse como comunidades inteligentes, capaces de pensarse a sí mismas y de gestionar su conocimiento, complementando la docencia presencial con la virtual. Para que sean eficaces, como señalan Sánchez y Careaga (Medina y Badilla, 2013), es necesario asociar la inte- 
gración curricular de las TIC con nociones de Gestión del Conocimiento.

De acuerdo a esta investigación, y es similar al estudio anterior, ya que, al permitir establecer mejor uso de las tecnologías de información, se obtiene una mejor calificación en los contenidos dictados en clase, así mismo, al realizar un aula virtual de aprendizaje mejora el rendimiento de los estudiantes, complementándolo con el desarrollo de las habilidades cognitivas para dar respuestas especificas a los problemas presentados durante el proceso de enseñanza-aprendizaje mediante el fortalecimiento de las competencias conceptuales (Aparicio y Ostos, 2018).

Por su parte, Fantinni, Caraballo, Cucci, Ferrante, y Graieb (2014), en su trabajo denominado: La integración de las TIC en las aulas de Ciencias Naturales. Experiencias de "Escuelas de Innovación". En donde mencionan que, en las últimas décadas, se ha producido en la Argentina y en el mundo un cambio sociocultural fundamental que involucra la incorporación de las TIC (Tecnologías de la Comunicación y la Información) y otras tecnologías en las diferentes actividades humanas. La vida escolar no es ajena a este contexto de incorporación de nuevas tecnologías y la integración de recursos tecnológicos le afecta desde diversos puntos de vista. Los jóvenes tienen una nueva manera de relacionarse con la tecnología y sus usos. Al menos en contextos urbanos, utilizan celulares y otros dispositivos que llevan a clase y cuya presencia produce una gran variedad de respuestas en los docentes, que van desde la prohibición de uso a la utilización con fines educativos.

Se puede destacar como resultados que: Los docentes muestran un gran cambio de actitud entre los primeros momentos del primer encuentro presencial y las capacitaciones subsiguientes. Si bien al comienzo algunos profesores se muestran reticentes y cautelosos frente al uso de tecnología y a la propuesta didáctica, en poco tiempo se los ve relajados y cómodos en la modalidad de trabajo, se entusiasman en los momentos de clase y las capacitaciones suelen transcurrir en un clima distendido y descontracturado. Igualmente, los profesores suelen apropiarse del material 
que se les entrega y con el que trabajan en las capacitaciones, llevándolo a sus propias aulas adaptado para su contexto y con su enfoque personal. Esto implica la incorporación de tecnología en el aula y, muchas veces, la incorporación de innovaciones pedagógicas en la enseñanza, la cual, como se ve, es facilitada por la integración de las TIC.

Por último, la aplicabilidad de este estudio resultó ser importante para la presente investigación, ya que, las actividades y modalidades de enseñanza y aprendizaje se ven afectadas por los planteamientos de nuevas y renovadas ideas y paradigmas y por la experiencia que, en materia de tecnología de la información, poseen estas nuevas generaciones de estudiantes. Las TIC ocupan un lugar en la vida escolar. Por acción u omisión, por haberse convertido en una molestia o por haber encontrado usos didácticos potentes, están ahí y no pueden ser ignoradas, pero su uso didáctico no necesariamente es evidente y debe estudiarse. En este sentido, dado que la tecnología está aquí para quedarse, tal vez es más adecuado investigar y experimentar en la manera de utilizarla con fines educativos, más que preguntarse si corresponde o no que estén presentes en la escuela.

\section{Antecedentes nacionales}

Para los antecedentes nacionales se tomó como referente la tesis elaborada por Morales Rojas Luisa Fernanda (2010) el cual tituló: "Leer para construir" : Proyecto de animación y promoción de lectura en los estudiantes de quinto Grado del Gimnasio campestre Beth Shalom cuyo objetivo principal fue: Sensibilizar a los estudiantes y profesores del Colegio Campestre Beth Shalom sobre la importancia y sensibilidad de la lectura en los procesos de aprendizaje a través de la propuesta didáctica, Programa de Promoción y Animación a la lectura, utilizó una metodología de tipo descriptivo en la que se concluyó que el aporte principal que se puede tomar de este trabajo es que los resultados de análisis de la información recolectada permitieron evidenciar que la escuela, la familia y la biblioteca tanto pública como escolar están en 
la obligación de formar y fortalecer los hábitos y la competencia lectora en los niños.

La aplicabilidad de este trabajo se torna interesante, debido a que la lectura de este trabajo nos permitió afianzar la idea de que a través de la aplicación de una buena estrategia se puede despertar el hábito hacia la lectura no como algo repetitivo, sino como una actividad fructífera y anímica, que lo va a llevar a enriquecer su propio conocimiento y lo oriente a realizar lecturas recreativas y voluntarias, apropiándose críticamente de ellas (de Almeida, Santos, Porto, 2017).

Igualmente, García (2015), menciona en su investigación "Metodologías didácticas para la enseñanza y aprendizaje de las Ciencias Naturales en zonas rurales del municipio de Obando, Valle del Cauca. Trabajo final de interpretación cualitativa, que tomó principalmente elementos metodológicos del estudio de caso y la investigación por encuesta, utilizados para analizar las metodologías implementadas actualmente para la enseñanza de las Ciencias Naturales, en los colegios oficiales de la zona rural del municipio de Obando en el Valle del Cauca, junto con su impacto en la población estudiantil. En lo referente a las metodologías didácticas para la enseñanza y aprendizaje de las Ciencias Naturales, aplicadas en la zona rurales del municipio de Obando, Valle del Cauca. De la investigación se puede concluir, que son principalmente de corte tradicional, con escasa aplicación de estrategias didácticas innovadoras, problematizadoras e investigativas, tendientes a la acumulación de conocimientos, y poco favorables para el desarrollo de competencias.

Al analizar lo manifestado por los docentes, se puede concluir que éstos consideran que la enseñanza de las Ciencias Naturales presenta serias problemáticas, por la dificultad para comprender los temas que allí se trabajan, la falta de motivación o desinterés de los alumnos, junto con la carencia de recursos idóneos para superar estos obstáculos.

En este sentido, la investigación aporta al estudio, una herramienta pedagógica esencial, que permite que los educandos lo- 
gren ampliar sus conocimientos, en este caso sobre las Ciencias Naturales y el medio ambiente y adquieran conciencia de su entorno, además puedan realizar cambios en sus valores, conductas y estilos de vida, para promover los procesos de prevención y resolución de problemas ambientales.

Desde estos aspectos, resulta crucial que en los centros educativos fomenten las buenas prácticas educativas con relación a las Ciencias Naturales y la Educación Ambiental, donde la predominancia de métodos de enseñanza de tipo tradicional, sin iniciativa a mejorar y ser más, es el principal obstáculo que limitan el desarrollo de metodologías más significativas de la enseñanza; por otra parte, esta situación, ha generado apatía y falta de motivación hacia la escolarización. Recomendándose un proceso de socialización y reflexión en torno a diversas estrategias y alternativas encaminadas hacia la transformación de la práctica docente y la interacción con los estudiantes, aprovechando el contexto rural y orientado hacia la generación de procesos más dinámicos, en pro de la motivación del estudiantado y el aprendizaje significativo.

Finalmente, Blancas y Rodríguez (2013), en su proyecto "Uso de tecnologías en la enseñanza de las ciencias. Caso de una maestra de Biología de secundaria" En este trabajo se presenta, desde una perspectiva situada y cualitativa, una descripción de las formas en que una maestra de Biología de educación secundaria usa algunas herramientas tecnológicas en su trabajo en el aula. El análisis descriptivo permite caracterizar la práctica docente de la maestra, así como identificar matices en el uso de las herramientas. Los hallazgos indican una práctica donde las herramientas tecnológicas se mezclan con ciertas relaciones y estilos de enseñanza centrados en la transmisión de información. El trabajo responde a la necesidad, en el contexto de la Reforma a la Educación Secundaria en México, de realizar estudios empíricos que indaguen las dimensiones del cambio en la enseñanza de las ciencias a través de la incorporación de tecnologías. Los resultados del trabajo permiten discutir aspectos relevantes cuando se trata de dar cuenta de qué es lo cambia, o no, cuando se incorporan tecnologías en las prácticas de enseñanza. 
Se concluye que en este trabajo se describen las formas en que una maestra de Biología de educación secundaria, usa herramientas tecnológicas multimedia interactiva y animación en flash, articulados en un software para instalar en la computadora en su práctica de enseñanza. La descripción de los usos observados pone en evidencia la realización de actividades radicalmente poco innovadoras, es decir, la presencia de dichas herramientas parece no modificar la práctica de enseñanza. Hecho que no concuerda con las expectativas de renovación de las prácticas de enseñanza al incorporar tecnologías que subyacen en la retórica del discurso oficial.

Para finalizar en el ámbito nacional, se tiene que el aporte que deja la investigación es que la estabilidad educativa permanece en la práctica, solo que ahora las herramientas tecnológicas se fusionan con formas y estilos de enseñanza consideradas como tradicionales del mundo escolar. Lejos de las grandes transformaciones que se anticipan en la retórica pedagógica oficial sobre la incorporación de tecnologías en la enseñanza de las ciencias, los ejemplos de los usos de las tecnologías identificados en esta investigación denotan, de manera general, relaciones pedagógicas consideradas como verticales y, a su vez, prácticas escolares bastante conocidas y arraigadas en las prácticas escolares, las cuales privilegian procesos de transmisión de información así como la promoción de destrezas técnicas y aisladas.

\section{Antecedentes locales}

A nivel local es importante tener en cuenta las siguientes investigaciones:

Molina (2011) elaboró su tesis de grado "Refinamiento y profundización del conocimiento en la comprensión lectora " presentada a la Universidad Industrial de Santander (UIS) en la facultad de ciencia humanas de la escuela de educación en maestría en pedagogía en Bucaramanga, cuyo objetivo es implementar una propuesta de fortalecimiento para la comprensión lectora que potencialice el de- 
sarrollo de operaciones cognitivas y presente una propuesta para el refinamiento y profundización del conocimiento con estudiantes de segundo semestre de la escuela de trabajo social de esta misma institución.

La metodología implementada permitió reflexionar sobre temas que hacen que el estudiante hile lo teórico con lo práctico, establezca comparaciones, contrastaciones, análisis de posturas teóricas, discusiones y lecturas complementarias necesarias para comprender los textos técnicos. Concluyendo, en esta propuesta el objetivo se fundamenta en la investigación de un proyecto de fortalecimiento para la comprensión de lectura que potencialice el desarrollo de operaciones cognitivas y posibilite el refinamiento y profundización del conocimiento.

Otro aspecto destacado es que la responsabilidad de comprender y leer no es función netamente de los estudiantes, sino que debe ser compartida entre estudiantes, profesores e instituciones. No es la cantidad ni la complejidad de los textos básicos la que garantiza el aprendizaje de los educandos, sino que en el incluyen también las estrategias y metodologías que insisten en el planeamiento de las asignaturas y la claridad de propósitos educacionales presentes en el proyecto pedagógico de la institución.

En concordancia, la investigación en el ámbito local deja un aporte particular al estudio en curso, ya que, los estudiantes participantes percibieron este proceso como muy novedoso e indispensable, lo que quiere decir que al aplicar estrategias innovadoras no solo mejora el rendimiento académico, sino que incentiva a los estudiantes a familiarizarse con contenidos teóricos que suelen ser desmotivadores e incomprensibles. La evaluación permanente en el mismo hizo evidente su pertinencia para el logro de los objetivos de aprendizaje.

Por otra parte, Hernández (2010), investigador de la Universidad Industrial de Santander (UIS), diseñó el proyecto "Sistema virtual de lectura como apoyo al mejoramiento de la comprensión lectora orientado a estudiantes de último grado de educación básica pri- 
maria en el municipio de Bucaramanga" su objetivo se centró en determinar la influencia en el comportamiento lector que ejerce un sistema de apoyo al mejoramiento de la lectura en alumnos de último grado de educación básica primaria en el colegio La Juventud del municipio de Bucaramanga. La metodología que utilizó primero la enfocó al estudio de las escuelas en cuanto a su naturaleza (oficial, privada) ubicación y otros factores sociales y de acceso a la información como el Internet.

Después se realizaron pruebas diagnósticas, para analizar el comportamiento de los estudiantes al documentarse e implementar la propuesta y realizar recomendaciones que se ajustaran a la estrategia planteada, a través de la implementación de talleres de lectura en el área de informática con el grupo experimental y su vez paralelamente implementación de talleres de apoyo en el aula cuyo objetivo pretendía nivelar y mejorar la comprensión lectora, luego la evaluación comparativa de los dos grupos para formalizar el sistema de apoyo al fortalecimiento lector.

Con la implementación de esta propuesta se logra desarrollar una empatía del manejo de las TIC para mejorar la comprensión lectora. Al finalizar este proyecto se concluyó que la educación virtual se está convirtiendo en una alternativa de educación y no en moda, por ello se le debe dar el tratamiento que exige cualquier ambiente de aprendizaje que motive al alumno hacia los textos, apoyándose con las herramientas que brinda un sistema virtual de aprendizaje donde se favorezca la relación del alumno con la lectura y el texto.

En otras palabras, se deduce que este trabajo es muy positivo ya que trabaja mancomunadamente en el proceso de leer y comprender para que sea una tarea responsable de estudiantes, profesores y comunidad educativa en general, que incluya en su proyecto institucional estrategias que sean direccionadas a la formulación de procesos de comprensión lectora enriquecedora y productiva para todos (Ferreyra, 2014). 


\section{Diseño metodológico}

\section{Tipo de investigación}

En cuanto al método utilizado en este proyecto, se considera apropiado la investigación acción que, según Hernández, Fernández, \& Baptista (2010), la define como "una actividad auto-reflexiva realizada por el profesorado con la finalidad de mejorar sus prácticas" (p.9). Con base en esta postura, se puede decir que los docentes a diario deben analizar sus prácticas de aula, tendientes a reflexionar sobre cada uno de los procesos ejecutados con el fin de innovar, aplicar nuevas estrategias, que conlleven a desarrollar conocimientos transformadores a través de la relación con el estudiante y así poder contribuir a la eficacia de la propuesta planteada, este proceso se hará de una forma cualitativa.

Según Mckernan (1999), describen la investigación acción (IA) como aquello que "Se construye desde y para la práctica, (ii) pretende mejorar la práctica a través de su trasformación, al mismo tiempo que procura comprenderla, (iii) demanda la participación de los sujetos en la mejora de sus propias prácticas" (p.2). De acuerdo a su afirmación, se puede establecer que en la investigación acción participativa juega un papel fundamental el entorno social para la resolución de problemas cotidianos. En otras palabras, la IA interpreta el comportamiento de los estudiantes de grado octavo en la asignatura de Ciencias Naturales y Educación Ambiental para darle solución al sistema educativo.

Por su parte, la investigación respondió al estudio no experimental, de tipo descriptivo, ya que la información recabada coincide con el tipo de propuesta que existe en el currículo escolar de las Ciencias Naturales con relación a los contenidos conceptuales y procedimentales, además, se relaciona con la forma en que los estudiantes conciben los conocimientos para darle solución a los distintos problemas que se les presenta en su entorno social.

La aplicación de aprendizaje de las Ciencias Naturales y la Educación Ambiental en los estudiantes de grado octavo aborda la 
enseñanza de los contenidos procedimentales desde una visión descriptiva. Por ello, Hernández, et al. (2010) expresan que la investigación de tipo descriptiva se asocia con la caracterización de una situación en particular para analizar sus causas y consecuencias.

Por su parte, el estudio en curso es considerado de tipo descriptivo porque intenta especificar las propiedades, las características y los diversos aspectos, dimensiones o componentes del fenómeno a investigar. La información obtenida de los estudiantes de grado octavo sirvió como parámetro para delimitar los contenidos procedimentales a incluir, en el instrumento aplicado, a la muestra objeto de estudio para diseñar las unidades didácticas utilizadas durante la intervención.

De acuerdo con lo anterior, la investigación es longitudinal. Según, Hernández et al (2010), explican que los diseños longitudinales, son los que representan datos a través del tiempo en puntos o periodos, para hacer inferencias respecto al cambio sus determinantes y consecuencias.

Este proyecto se inició con la observación directa a los estudiantes en las diferentes actividades que se desarrollan en el aula y así constatar las falencias que presentan en el proceso de la comprensión teórica en los contenidos de Ciencias Naturales y Educación Ambiental. Mediante un Análisis Documental, con base a las diferentes evaluaciones internas que se realizaron antes y después de la ejecución de la implementación de las estrategias pedagógicas para mejorar la comprensión teórica en la asignatura de Ciencias Naturales y Educación Ambiental. Posteriormente se hizo un estudio sobre los resultados obtenidos en las pruebas PISA y Saber, dónde se identificaron los ítems de desempeño que se tuvieron en cuenta para el proceso de mejoramiento y fortalecimiento de la comprensión teórica de los contenidos conceptuales en el área de Ciencias Naturales y Educación Ambiental.

Para el Diseño de esta propuesta se tomaron en cuenta los lineamientos pedagógicos de la didáctica y las teorías del aprendizaje significativo de Ausubel (1968) y constructivista de Piaget 
(1976). Para la aplicación de la propuesta educativa se escogió el contenido de las células, que corresponde a la asignatura de Ciencias Naturales, base de contenidos más complejos como son la formación de células hematopoyéticas. Como parte de esta fase, se construyó un entorno virtual de aprendizaje, el cual se diseñó con el fin de que los estudiantes pudieran acceder a cada una de las unidades didácticas estructuradas en el portal educativo SMORE.

La fase de diseño estuvo estructurada por 4 unidades didácticas:

Unidad I: La célula : https://www.smore.com/3vsca Unidad II: Tipos de células: Reconozco las diferencias entre clases y tipos de células. https://www.smore.com/ typre

Unidad III: Tipos de células: eucariotas y procariotas. https://www.smore.com/qydyx

Unidad VI: La célula: partes y orgánulos https://www. smore.com/rp3kn

Seguidamente, la Implementación de esta propuesta se realizó en cuatro semanas.

Cada unidad temática se desarrolló bajo la capacidad de comprensión teórica de cada uno de los participantes. En esta fase se realizaron una serie de actividades que permitieron registrar el rendimiento académico de cada educando, para conocer el avance de los estudiantes, se utilizó una rúbrica de evaluación para cada actividad realizada, instrumento que permitió registrar detalladamente cada una de las estrategias implementadas durante la implementación de la propuesta.

Las rubricas de evaluación se usaron como guías para conocer el nivel de motivación de los estudiantes sobre las actividades realizadas. En este instrumento se evaluaron las subcategorías como: implementación de herramientas web 2.0., motivación, participación activa, componente cognitivo y componente procedimental, con el fin de abordar las características específicas motivacionales que intervienen en el desempeño de las competencias académicas. 
La elaboración de las rubricas de evaluación para cada actividad permitieron hacer una reflexión sobre la práctica pedagógica del docente y realizar a la vez una categorización y subcategorización de los hallazgos encontrados llevándolo a replantear procedimientos innovadores tendientes a fortalecer las dificultades identificadas. A su vez al analizar las observaciones realizadas y los resultados de las diferentes pruebas externas propuestas por el Estado se hizo una confrontación donde se estableció la estrategia a utilizar en beneficio de la problemática planteada.

Por medio del análisis documental se pudo plantear acciones para clarificar que el modelo pedagógico del colegio es el constructivista social pero al interactuar con los procesos de observación y el resultado obtenido en cada actividad se llegó a la conclusión que no todas las actividades se orientan a las características de este modelo; es pertinente tener en cuenta los aportes de los teóricos que lo representan para hacer una intervención en las necesidades encontradas, diseñando una propuesta pedagógica a través de las secuencias textuales plasmadas en proyectos de aula.

\section{Población y muestra}

\section{Población}

Flores (2004), definen a la población como "el conjunto de todos los individuos (objetos, personas, eventos) en los que se desea estudiar el fenómeno. Éstos deben reunir las características de lo que son objeto de estudio". Para su selección se hizo necesario contar con algunos elementos, como la homogeneidad, donde se observaron los mismos rasgos de acuerdo con la variable considerada en la investigación. A su vez, se debió tomar en cuenta el tiempo donde se ubicó esta población y el espacio, que no fue más que el lugar donde se encontraron los individuos a investigar.

La población se desarrolló en el Instituto Politécnico de Bucaramanga, la cual estuvo conformada por 145 estudiantes que cumplen sus jornadas académicas en la tarde. De esta población se 
extrajo una muestra intencional de 35 estudiantes que conforman el grado de octavo, en las edades de 13 a 14 años.

\section{Muestra}

Al hablar de muestra, los autores Valenzuela \& Flórez (2012), la definen como "un subgrupo de esa población que debe ser representativo de la misma, se extrae cuando no es posible medir a cada una de las unidades de la población" (p. 240). Por lo tanto, una muestra es un conjunto de unidades, una porción del total, que representa la conducta del universo en su conjunto, en un sentido amplio, no es más que una parte del todo que se llama universo o población y que sirve para representarlo. De esta población se extrajo una muestra intencional de 35 estudiantes que conforman el grado de octavo, en las edades de 13 a 14 años del I.E. Técnico Politécnico de Bucaramanga, Santander.

\section{Instrumentos para la recolección de la información}

Para la realización de este proceso se tuvo en cuenta algunos instrumentos que permitieron recoger información, los cuales se relacionan en la siguiente tabla:

Tabla 1. Instrumentos para la recolección de la información. Fuente. Elaboración propia.

\begin{tabular}{|l|l|l|}
\hline Técnicas de recolección & Técnicas de registro & Dirigido a quien \\
\hline Observación Participativa & Observación & Estudiantes \\
\hline Análisis documental & Ficha de registro & Estudiantes \\
\hline Rubricas de Evaluación & Guías de Pruebas & Estudiantes \\
\hline
\end{tabular}

Observación Participativa. Es la más apropiada para obtener resultados de alta confiabilidad ya que el autor de esta tesis se encuentra involucrado directamente en el quehacer pedagógico, y 
para ello Mckerman (1999) la define "como la práctica de hacer investigación tomando parte en la vida del grupo social o institución que está investigando. Así el investigador tiene una meta doble: asumir el rol de un participante e investigar el carácter etnográfico del entorno" (p. 84).

Análisis Documental. Es un instrumento de investigación para analizar documentos institucionales, informarles que permita saber cómo está la situación problema a estudiar, a dónde queremos llegar y sobre todo como lo vamos a conseguir. La calidad educativa no se limita solamente al cumplimiento con las normas establecidas con el MEN ni a los resultados de las pruebas estandarizadas sino a un análisis profundo y a la puesta en marcha de un plan de mejoramiento eficaz.

Rúbrica de Evaluación. Las rúbricas han emergido como un instrumento valioso para la evaluación de competencias. Según, Blanco (2008), pese a su interés, no pueden resolver todas las dificultades asociadas a los procesos de evaluación. Una rúbrica es, como se ha indicado, un registro evaluativo que posee ciertos criterios o dimensiones a evaluar y lo hace siguiendo unos niveles de calidad tipificando los estándares de desempeño. Este tipo de instrumento se usa como guías para conocer el nivel de motivación de los estudiantes en los conocimientos teóricos. En este instrumento se evaluará a través de las categorías, estrategias pedagógicas y compresión teórica, que intervienen en el desempeño de las competencias académicas.

Tabla 2. Categorías y Subcategorías. Fuente. Elaboración propia.

\begin{tabular}{|l|l|}
\hline Categorias & Subcategorias \\
\hline Preparación de clases & Motivación, planificar, desenvolvimiento, interés, actitudes \\
\hline Prácticas de aula & Contextualizar, interactuar, descubrir e innovar \\
\hline Evaluación de los aprendizajes & $\begin{array}{l}\text { Texto, aprendizaje, narración, analizar, confrontar, construir y } \\
\text { socializar }\end{array}$ \\
\hline Discursos en el aula & Comunicación, sentimientos, sensibilizar, valorar \\
\hline
\end{tabular}




\section{Propuesta pedagógica}

Como parte de la fase de diseño, se usó el portal SMORE para registrarse y poder usar esta herramienta web 2.0, para el diseño y construcción de los talleres concernientes a cada una de las unidades didácticas de la investigación.

En este caso, se prefirió usar SMORE ya que es una herramienta Web 2.0 de fácil manejo y de modo público con el fin de que los estudiantes no tuvieran necesidad de registrarse, sino que sólo a través de un link pudieran acceder de manera ágil a cada uno de los talleres virtuales construidos sobre el contenido de las células.

La figura siguiente muestra el perfil SMORE que el autor de esta investigación uso para fortalecer la comprensión teórica de contenidos conceptuales en el área de Ciencias Naturales y Educación Ambiental. Acceso Directo a la actividad: https://www.smore. com/3vsca

\section{Resultados}

Para efectos de la presentación de los resultados se hizo una organización a partir de las categorías particulares a las que subyacen unas subcategorías que expresan los procesos de práctica realizadas en el grado octavo donde se llevó a cabo la investigación.

\section{Preparación de clases}

En la preparación de las clases de Ciencias Naturales y Educación Ambiental se escogió el tema de las células, como tema piloto para la implementación de las estrategias pedagógicas mediadas por las herramientas web 2.0, para fortalecer la comprensión lectora en los estudiantes de grado octavo en el I.E. Técnico Politécnico de Bucaramanga. Cada actividad planificada y desarrollada en clase estuvo sustentada con base en la Teoría del Aprendizaje Significa- 
tivo de Ausubel (1968) y la teoría constructivista de Piaget (1976). Ambas teorías demuestran que el profesor debe dejar de tener un papel protagonista en el proceso de aprendizaje, es decir, requiere entender que el nuevo paradigma educativo apunta al estudiante como el centro del proceso educativo. En este sentido, El aprendizaje pasa a ser un problema del profesor; "lo importante no es transmitir, sino ayudar al alumno a adquirir conocimientos y a desarrollar su capacidad de reflexión y comprensión." (Rodríguez E. L., 2009).

Consecuentemente, durante el desarrollo de la investigación acción se organizó y gestionó la preparación de las clases con objetivos claros y estrategias didácticas. Así, la realización de las actividades de aprendizaje propiciaba el gran potencial en los estudiantes en torno a las competencias de lectura y escritura.

Sin duda, para la preparación de las clases en el grado de octavo se tuvo muy en cuenta un proceso de reflexión profundo, en el que, según Rodríguez E. L. (2009) involucra una serie de factores, tales como:

- Conocimiento del rumbo de la institución

- Conocimiento de la realidad social

- Conocimiento sobre los estudiantes

- Conocimiento sobre los niveles de la disciplina

Por ello, es posible destacar como resultados positivos en el desarrollo de la preparación de clases durante la investigación, el hecho de que los estudiantes no sabían con certeza, cuál era el motivo de su aprendizaje, dicho de otro modo, se procuró siempre que el mismo educando descubriera que el proceso de aprendizaje no era arbitrario, sino que, por el contrario, tenía una significancia cotidiana, social, cultural, escolar, etc. (Aparicio, 2018).

\section{Prácticas de aula.}

Según la Teoría del aprendizaje significativo de Ausubel, el aprendizaje depende de las motivaciones, intereses y predisposición del 
aprendiz, además de los conocimientos previos y los nuevos por construir. De esta forma, el educando "no puede engañarse a sí mismo, dando por sentado que ha atribuido los significados contextualmente aceptados, cuando sólo se ha quedado con algunas generalizaciones vagas sin significado psicológico" (Rodríguez, 2004, p. 16).

Por esta razón, las prácticas de aula realizadas en la investigación y durante la implementación de las estrategias pedagógicas constructivistas y significativas, correspondieron con las formas en la que los estudiantes se sentían mayormente motivados, de esta manera, la puesta en marcha de actividades con ayuda de herramientas tecnológicas tales como proyecciones de videos y actividades interactivas, permitió que los aprendientes se sintieran motivados y al mismo tiempo conscientes de que se trataba de un proceso de aprendizaje continuo y significativo (Viejo, Cabezas, Martínez, 2013).

En consecuencia, la propuesta fue realizada durante cuatro semanas, a través de las siguientes unidades temáticas, las células, clases de células, tipos de células y por último funciones y estructuras de las células. Cada una de ellas se evaluaron las subcategorías contextualizar, interactuar, descubrir e innovar.

Durante la implementación de las unidades temáticas se observó que el aprendizaje teórico requiere de estrategias que acompañen el aprendizaje de los estudiantes, al contextualizar los contenidos de las células, los estudiantes expresaron que aprender con las TIC facilitaba e incentivaba el conocimiento que ellos deben adquirir durante el proceso de la enseña y aprendizajes.

Por su parte, cada una de las actividades realizadas, se llevaron a cabo mediante la convicción de que es necesario indagar en los contenidos antes de mostrarles a los estudiantes las actividades interactivas, ya que al no comprender dichas actividades el recurso educativo digital puede llegar a convertirse en un factor distractor para los mismos, ocasionado una situación tensa tanto para el grupo como para el docente. A su vez, se concientizó al estudiante de 
que los contenidos eran la base para actividades futuras a realizar, las cuales abordaban conceptos más complejos como son la formación de células hematopoyéticas.

En este sentido, se requiere indagar cómo se promueve efectivamente el aprendizaje significativo y cómo se construye el conocimiento en las aulas, qué factores facilitan estos procesos y qué factores los dificultan. En efecto, una de las consecuencias de las buenas prácticas de aula fue el aprendizaje constructivo enunciado por Piaget (1976), en el que a través de la participación activa en clase se genera un proceso de autorreflexión, en el que el propio aprendiente es capaz de dirigir la manera en la que aprende (Rivero, 2009). En definitiva, las prácticas de aula surgieron como ambientes de aprendizaje apropiados, puesto que los educandos no se sentían en clase de contenidos teóricos, largos y convencionales, sino más bien en un proceso de enseñanza-aprendizaje dinámico y continuo mediante el contenido de las células y los tipos de células.

\section{Discurso de aula}

En esta categoría, se encontró una estrecha relación entre el discurso docente y la preparación de clase, en la medida en que, si se poseen los objetivos de aprendizaje claros para cada actividad diseñada, se generarán procesos de orientación oral por parte del docente de manera más clara y concisa. En este sentido,

La acción de rediseñar la clase se convierte en una reflexión teórica y metodológica de la acción educativa, por lo tanto, requiere que el maestro aprenda, comprenda y elabore un plan sobre qué, para qué, por qué y cómo realizar el proceso de enseñanza-aprendizaje (Rodríguez, 2009, p. 21).

Consecuentemente, durante la ejecución de las estrategias pedagógicas el docente de la asignatura, en sus discursos de aula, debía ser un mediador de los aprendizajes de los estudiantes para 
lograr que la comunicación fluyera en un ambiente de confianza y seguridad que construya habilidades para formar una plena autonomía y promover sentimientos positivos, en cuanto a despertar intereses para alcanzar nuevas metas, así, se conformaba lo que Dewey denominaba una mini comunidad de vida (Dorantes \& Matus, 2007).

Es importante resaltar que la postura constructivista adoptada en esta investigación facilitó que, el aprendizaje fuera realmente significativo en los estudiantes de grado octavo de la I.E. Técnico Politécnico de Bucaramanga. El contenido de ciencias naturales como las células se utilizó como programa piloto para verificar si la propuesta daba resultados positivos en los estudiantes de grado octavo.

Por su parte, la postura constructivista del aprendizaje sostiene que toda actividad mental es constructiva, de tal manera, que los estudiantes de octavo grado adquirieron los nuevos conocimientos a través de un proceso activo e interactivo de asimilación y acomodación, donde tanto lo nuevo como lo ya existente se transforma a medida que el alumno construye esquemas de comprensión más inclusivos, mejorando así el proceso de la comprensión lectora, dándole sentido a todo lo que él aprende vinculándolo con su entorno.

En definitiva, orientar prácticas de aula dinamizadoras e innovadoras facilitó a los estudiantes confrontar nuevos aprendizajes para aclarar dudas y aprovechar sus dificultades para promover conocimientos construidos a través de sus pre saberes con el intercambio de situaciones significativas.

\section{Evaluación de los aprendizajes}

Dewey menciona que se debe dejar de pensar en las materias de estudio como algo rígido y ya elaborado por sí mismo, fuera del estudiante. En este ámbito, el proceso de aprendizaje se debe observar como: 
...algo que fluye, vital y así se comprenderá que el niño y el programa son solamente dos límites que definen un solo proceso. La instrucción no es más que una continua reconstrucción de la experiencia que va del niño a las concepciones de verdad organizadas, llamadas materias de estudio. (Dorantes \& Matus, 2007, p. 4)

Desde esta perspectiva, hacer un seguimiento de los aprendizajes de los estudiantes y evaluar cada una de las unidades temáticas utilizadas, fue importante para analizar las dificultades en la construcción de los conocimientos a través de la comprensión teórica, pero no de la forma convencional, sino procurando que el alumno se autoevaluara a sí mismo y de forma grupal, para que permita una socialización respetuosa con sus compañeros, reconociendo así aspectos de mejora para que la evaluación no se tratase solamente de una herramienta calificadora, sino motivadora. En este orden de ideas, se hizo uso de distintos instrumentos de evaluación tales como la evaluación formativa y la evaluación continua, las cuales se apoyaban en las competencias que el estudiante iba adquiriendo en el proceso de enseñanza-aprendizaje sobre la comprensión teórica en los contenidos de Ciencias Naturales y Educación Ambiental.

Los aprendizajes de los estudiantes fueron evaluados a través de la utilización de las rubricas de evaluación enfocadas a revisar el mejoramiento de los estudiantes en las actividades realizadas durante la aplicación del programa, sin embargo, este instrumento también permitió hacer una autoevaluación del quehacer pedagógico del docente, logrando identificar los ajustes necesarios a aplicar en sus prácticas en aras de mejorar el desempeño en el aula, en busca de estrategias que sean benéficas para mejorar la problemática encontrada.

De esta manera, durante la unidad I se observó que los estudiantes al principio se extrañaban por participar en una clase más dinámica e interactiva, expresaban que lo normal era que el docente dictará la clase desde el tablero, asignará algunas preguntas y luego evaluará los contenidos. Sin embargo, al ingresar al 
aplicativo web los estudiantes empezaron a sentirse interesados con la presentación del video sobre las células. El resultado fue gratificante para el autor de la propuesta porque la participación y el trabajo colaborativo arrojo un alto aprendizaje, esta unidad fue evaluada con una rúbrica de evaluación donde se cumplió el primer objetivo de esta propuesta el cual consistió en desarrollar habilidades en la comprensión lectora.

Sin embargo, a través de la técnica de observación se evidenció que los estudiantes se sentían animados realizando la unidad II "clases de células". Durante esta semana se presentó un cambio positivo y más ameno en la visualización del video sobre la célula vegetal y la célula animal, la experiencia fue totalmente contario a la primera semana, para los estudiantes ya era familiar utilizar el recurso digital educativo, expresaban que las clases deberían estar asociadas a las tecnologías educativas porque no solo ayudaban al aprendizaje de los contenidos, sino también, que ayudan a mantener el orden y la disciplina en el aula haciendo más gratificante la clase.

En este mismo orden de ideas, la unidad III y IV permitieron realizar escritos en el aula y sopa de letras con palabras relacionadas con los orgánulos celulares a través de la observación de los videos, además presentaron de forma oral lo que comprendieron de las células, evaluándose que el nivel de comprensión teórica en los estudiantes grado de octavo mejoró notoriamente. Se evidenció que este tipo de actividades deja resultados positivos para la investigación, igualmente se convierte en un elemento importante para mejorar el rendimiento académico de los estudiantes en las distintas áreas del aprendizaje.

Es importante resaltar que las subcategorías comunicación, sentimientos, sensibilización y valoración, permitieron hacer las reflexiones del estudio sobre algunos de los componentes del PEI entre ellos el pedagógico donde se pudo establecer cuál era su enfoque, modelo pedagógico que es el constructivista social, misión y horizonte institucional. La prueba de medición externa nacional SA$B E R$ que establece las dificultades que presentaron los estudiantes 
en el área de Ciencias Naturales y Educación Ambiental en cuanto a la comprensión teórica y dan una posición de la institución a nivel nacional.

Finalmente, con respecto al análisis de los resultados finales obtenidos a través de las rubricas de evaluación, se encontró que los educandos mejoraron de forma significativa en las cuatro categorías evaluadas, en general, la categoría con mayores dificultades fue la práctica de aula, la cual se caracterizó por incidir en la dificultad de los estudiantes por extraer conclusiones o hipótesis en base a la información implícita presentada o descrita en el contenido de las células. Sin embargo, en este caso, el desempeño de los estudiantes de la muestra de investigación fue de un nivel alto, debido principalmente a que el promedio global de resultados fue de 4.4 , hecho que demuestra que los estudiantes dominan de forma general la competencia de comprensión teórica con sus diferentes categorías.

En conclusión, el nivel alto de la muestra permitió inferir que los estudiantes del grado octavo son capaces de entender textos cortos de manera apropiada, definiendo sucesos que categorizan el inicio, desarrollo o cierre de los contenidos según el área de aprendizaje. Por ende, es posible afirmar que la aplicación de estrategias pedagógicas, permite mejorar la forma cómo los estudiantes se apropian del proceso y comprensión de los contenidos teóricos y construyen conocimiento, en este caso, dinamizándose las falencias presentadas de esta forma en el proceso de enseñanza y aprendizaje escolar.

\section{Conclusiones}

Es necesario expresar que el concepto de comprensión teórica presenta múltiples estereotipos de negatividad, desmotivación, aburrimiento entre otras características que los estudiantes le agregan a la hora de aprender asignaturas teóricas, como son Ciencias Sociales, Ética y valores, Ciencias Naturales y la Educación Ambiental. Por esta razón, la investigación busca responder la siguiente inte- 
rrogante ¿Cómo las estrategias pedagógicas constructivistas mediadas por las herramientas Web 2?0, contribuyen al fortalecimiento de la comprensión teórica de los contenidos conceptuales en el área de Ciencias Naturales y Educación Ambiental en estudiantes de grado octavo?

En consecuencia, para el desarrollo del primer objetivo específico de la presente investigación, se analizó la percepción de los estudiantes de octavo grado sobre las estrategias pedagógicas usadas en la enseñanza- aprendizaje de las Ciencias Naturales y Educación Ambiental, obteniéndose como conclusión que: la aplicación de estrategias pedagógicas constructivistas e innovadoras fortalece el entorno de enseñanza y aprendizaje, generando confianza en el estudiante y propiciando el mejoramiento de la disciplina en clase y el trabajo colaborativo en el aula.

Por otra parte, y teniendo en cuenta el segundo objetivo específico el cual era diseñar un entorno virtual de aprendizaje (EVA) mediante el uso de las herramientas web 2.0 para la enseñanzaaprendizaje de las Ciencias Naturales y la Educación Ambiental, permitió conocer que los estudiantes desarrollan mejor el proceso de aprendizaje a través de las herramientas tecnológicas, ya que, lo consideran innovador y fuera de la rutina diaria de la clase, haciendo más ameno el aprendizaje y la convivencia en el aula de clase.

También, se evidenció que, en la medida, en que se diversifique la manera como se enseña y se practica la lectura en el aula se mejora la comprensión de los contenidos teóricos a través de la compresión significativa de la lectura mediante la creación de hábitos de aprendizaje. Es importante destacar que la lectura es significativa para el proceso de enseñanza y aprendizaje, por lo que esta, va mucho más allá de la lectura automática, ya que la primera, involucra elementos lingüísticos, motores y psicopedagógicos. En este caso, se propició la lectura integral, es decir, a través de las actividades realizadas del contenido de las células implementada en el entorno virtual educativo, para que los estudiantes, fueran capaces de tomar una postura madura y diversificar su aprendizaje estableciendo características principales y secuencias dominantes, 
pero no necesariamente fragmentando el proceso de enseñanzaaprendizaje.

Así mismo, la implementación de un entorno virtual de aprendizaje (EVA) a través del uso de la web 2.0 permito fortalecer la comprensión teórica en el área de Ciencias Naturales y Educación Ambiental. Ya que la utilización de proyectores y herramientas de cómputo no garantiza una eficiente utilización de las TIC en el proceso de formación académica. Por lo tanto, se necesita un cambio de paradigma en el que la clase, realmente se componga de elementos digitales manipulados por el estudiante y que atiendan las necesidades del curso diseñado.

Finalmente, mediante la evaluación del impacto generado por el entorno virtual (EVA) de aprendizaje en los estudiantes del grado octavo del I.E. Técnico Politécnico Bucaramanga, se evidenció que es importante que se continúen realizando procesos de enseñanza-aprendizaje más enfocados en la llamada Escuela Nueva, en el Instituto Politécnico, ya que, estas acciones pedagógicas demostraron ser altamente eficientes para el mejoramiento de la calidad educativa dentro del aula.

En efecto, la asignatura de lenguaje debe continuar fomentando el gusto por la lectura a través de las secuencias textuales y la aplicación de métodos pedagógicos como los propuestos por M. Montessori (Silva \& Campos, 2003) y Dewey (Dorantes \& Matus, 2007), especialmente, porque se confirmó que ubicar al estudiante como centro de interés ocasiona el fortalecimiento efectivo de la comprensión teórica en los estudiantes.

\section{Recomendaciones}

Es importante tener en cuenta las siguientes recomendaciones:

- La conectividad a internet debe ser por lo menos de 1 GB para un correcto funcionamiento de portales como YouTube. 
- Es necesario que el docente tenga disponibilidad de tiempo para la búsqueda de material virtual actualizado y confiable. Además, es bueno verificar que en los videos utilizados no haya publicidad agresiva, religiosa, sexual o violenta que pueda desviar la atención de los estudiantes.

- Es necesario que se adecuen mejores formas de aprendizaje en torno a otras temáticas importantes, tales como la noticia, la crónica, los tipos de cuentos, etc. Ojalá, teniendo en cuenta, las secuencias textuales y la metodología de la Escuela Nueva, no para un periodo lectivo, sino para todos los del año escolar.

\section{Referencias bibliográficas}

Alcalá, G. (2012). Aplicación de un programa de habilidades meta cognitivas para mejorar la comprensión lectora en niños de cuarto grado de primaria del Colegio Parroquial Santísima Cruz de Chulucanas. Perú.

Aparicio, O.Y. (2018). Las TIC como herramientas cognitivas. Revista Interamericana de Investigación, Educación y Pedagogía, RIIEP, 11(1). https://orcid.org/0000-0003-3535-6288

Aparicio, O.Y. (2018). Las TIC como herramientas cognitivas para la investigación escolar. Revista Interamericana de Investigación, Educación y Pedagogía, RIIEP, 11(1). https://orcid.org/0000-0003-3535-6288

Aparicio, O.Y., \& Ostos, O.L. (2018). El constructivismo y el construccionismo. Revista Interamericana de Investigación, Educación y Pedagogía, RIIEP, 11(2). https://orcid.org/0000-0003-3535-6288. https://orcid.org/0000-0002-6477-9872

Ausubel, D. (1968). Teoría del Aprendizaje Significativo. Psicopedagogía Pedagogía y Psicología.

Barrantes, H. A., Beltrán, J. E. P., \& Pérez, F. A. R. (2016). Perfil del estudiante de pregrado de la Facultad de Estudios a Distancia de 
la Universidad Militar Nueva Granada. Revista Interamericana de Investigación, Educación y Pedagogía, RIIEP, 9(2). DOI: https://doi. org/10.22490/25391887.1948

Blancas, J., \& Rodríguez, D. (2013). Uso de tecnologías en la enseñanza de las ciencias. el caso de una maestra de biología de secundaria. Manizales: Universidad de Caldas.

Castro, A., \& Ramírez, R. (2013). Enseñanza de las ciencias naturales para el desarrollo de competencias científicas. Florencia, Colombia: Amazonia Investiga.

De la Calle, C. V., Malaver, M. O., Gallego, J. D. M., Rodríguez, M., Flórez, J. C., Henao, C. E. \& Saldaña, R. (2014). Aportes de los doctorados de educación en ciencia, tecnología y sociedad, desde la sistematización de sus investigaciones doctorales científicas y formativas, 2000-2010. Revista Interamericana de Investigación, Educación y Pedagogía, RIIEP, 7(1). DOI: https://doi.org/10.15332/s1657-107X.2014.0001.04

De Almeida, R. R., Santos, M. F., \& Porto, J. C. (2017). Lectura de textos ficcionales y el enfoque escolar de literatura: contribuciones para una Pedagogía de la Elección. Revista Interamericana de Educación, Pedagogía y Estudios Culturales, 9(1), 35-51. DOI: https://doi. org/10.22490/25391887.1925

Del Moral, B. C. (2010). Las secuencias textuales y la diversidad textual en la clase de L2: Propuesta didáctica para el aprendizaje autónomo mediante secuencias textuales. Revista Cervantes. Obtenido de http:// cvc.cervantes.es/ensenanza/biblioteca_ele/asele/pdf/21/21_1187.pdf

Dorantes, R. C., \& Matus, G. G. (2007). La Educación Nueva: la postura de John Dewey. Odiseo, revista electrónica de pedagogía. Obtenido de http://www.odiseo.com.mx/2007/07/print/dorantes-matus-dewey.pdf

Fantinni, V., Caraballo, D., Cucci, G., Ferrante, C., \& Graieb, A. (2014). La integración de las TIC en las aulas de Ciencias Naturales: Experiencias de "Escuelas de Innovación". Buenos Aires, Argentina: Congreso Iberoamericano de Ciencia, Tecnología, Innovación y Educación.

Ferreyra, H. A. (2014). Mesas Socioeducativas para la Inclusión y la Igualdad. Un programa "De todos con todos". Una experiencia en 
construcción. Revista Interamericana de Investigación, Educación y Pedagogía, RIIEP, 7(2). DOI: https://doi.org/10.15332/s1657107X.2014.0002.01

Gallego. (1997). Las estrategias cognitivas en el aula. Madrid.

Garcia, \& Magaz. (2000). Actualidad sobre el TDA-H.

García, S. (2015). Metodologías didácticas para la enseñanza y aprendizaje de las ciencias naturales. Palmira: Universidad Nacional de Colombia.

Hermosa Del vasto, P. (2015). Influencia de las tecnologías de información y comunicación (TIC) en el proceso enseñanza-aprendizaje: una mejora de las competencias digitales. Bogotá, Colombia: Revista Científica General José María Córdova.

Hernández, Y. (2010). Comprensión lectora y rendimiento académico en estudiantes de quinto de secundaria de una institución educativa del Callao. ISO 690. Callao.

Hernández, S. R., Fernández, C., \& Baptista, M. D. (2010). Metodología de la Investigación. México D.F.: The McGraw-Hill Companies.

Instituto Politécnico. (2012). Plan de Mejoramiento Institucional. Bucaramanga.

Konieczny, P. (2015). Lorenzo García Aretio: bases, mediaciones y futuro de la educación a distancia en la sociedad digital. Revista Interamericana de Investigación, Educación y Pedagogía, RIIEP, 8(1). DOI: https://doi. org/10.15332/s1657-107X.2015.0001.08

Leymonié, J. (2009). Aportes para la enseñanza de las ciencias Naturales. Santiago de Chile: Salesianos Impresores.

Mckernan, J. (1999). Investigacion Acción y Currículo. Madrid: Morata.

Medina, V., \& Badilla, G. (2013). Uso de ambientes virtuales en el aprendizaje de las Ciencias. Concepción -Chile: Universidad Católica de la Santísima. 
Ministerio de Educación Nacional. (2015). Estándares básicos de competencias en ciencias sociales y ciencias naturales. Bogotá. Obtenido de https://www.mineducacion.gov.co/1621/articles-116042_archivo_ pdf3.pdf

Ortiz, J. G., \& Buitrago, H. (2017). La evaluación en la tradición educativa colombiana. Instrumento de clasificación social. Revista Interamericana de Investigación, Educación y Pedagogía, RIIEP, 10(1), 145-171. DOI: https://doi.org/10.15332/s1657-107X.2148

Ospina, W. (2010). Carta al maestro desconocido.

Piaget, J. (1976). Teoría. Nueva York: Springer-Verlag.

Pósito, R. M. (2012). El problema de enseñar y aprender ciencias naturales en los nuevos ambientes educativos- tesis de maestría. Buenos Aires, Argentina: Universidad Nacional de la Plata.

Rivero, M. (2009). Teoría genética de Piaget: Constructivismo cognitivo. Universitat de Barcelona. Obtenido de http://diposit.ub.edu/dspace/ bitstream/2445/32321/6/Teoria\%20de\%20Jean\%20Piaget.pdf

Rodríguez, A. W. (1999). El legado de Vygotski y de Piaget a la educación. Revista Latinoamericana de Psicología, 477-489. Obtenido de http:// www.redalyc.org/pdf/805/80531304.pdf

Rodríguez, E. L. (2009). La planeación de clase: Una habilidad docente que requiere de un marco teórico. Odiseo, revista electrónica de pedagogía. Obtenido de http://www.odiseo.com.mx/2009/7-13/ rodriguez-planeacion_clase.html

Rodríguez, P. M. (2004). La teoría del aprendizaje significativo. Theory, Methodology \& Technology. Obtenido de http://cmc.ihmc.us/papers/ cmc2004-290.pdf

Silva, B. C., \& Campos, O. (2003). Método María Montessori. Psicología Educacional Valdebenito. Obtenido de http://www.elviajerosuizo.com/ resources/metodo.montessori-resumen.pdf

Valenzuela, J. R., \& Flórez, M. (2012). Fundamentos de Investigación Educativa. Editorial Digital Tecnológico de Monterrey. 
Viejo, C. M., Cabezas, I. L., \& Martínez, M. D. J. I. (2013). Las redes de académicas en la docencia universitaria. Revista Interamericana de Investigación, Educación y Pedagogía, RIIEP, 6(2). DOI: https://doi. org/10.15332/s1657-107X.2013.0002.03 